\title{
Features of Glucose Metabolism in Pleomorphic Hyalinizing Angiectatic Tumor (PHAT)
}

\author{
Po-Jui Chang ${ }^{1}$, Cher-Wei Liang ${ }^{2}$, Ching-Huei Kung ${ }^{3}$, Ching-Shui Huang ${ }^{4}$, Chung-Huei Hsu1* \\ ${ }^{1}$ Department of Nuclear Medicine, Taipei Medical University Hospital, Taipei, Taiwan \\ ${ }^{2}$ Department of Pathology, Cathay General Hospital, Taipei, Taiwan \\ ${ }^{3}$ Department of Radiology, Taipei Medical University Hospital, Taipei, Taiwan \\ ${ }^{4}$ Department of Surgery, Cathay General Hospital, Taipei, Taiwan \\ Email: ${ }^{\text {chhsu@tmu.edu.tw }}$
}

Received November 11, 2013; revised December 11, 2013; accepted December 18, 2013

Copyright (c) 2014 Po-Jui Chang et al. This is an open access article distributed under the Creative Commons Attribution License, which permits unrestricted use, distribution, and reproduction in any medium, provided the original work is properly cited. In accordance of the Creative Commons Attribution License all Copyrights (C) 2014 are reserved for SCIRP and the owner of the intellectual property Chung-Huei Hsu et al. All Copyright (C) 2014 are guarded by law and by SCIRP as a guardian.

\begin{abstract}
Pleomorphic Hyalinizing Angiectatic Tumor (PHAT) is a rare mesenchymal neoplasma. It is a pathological term describing a heterogeneous group of neoplasms of undetermined tumorigenesis and undefined malignancy. Based only on the morphological findings of histopathology, the biological potential of the tumor is indeterminate. Determination of glucose metabolism using FDG-PET revealed mildly increased FDG uptake in the tumor. We propose that FDG accumulation in the ectatic vessel, increased uptake of the pleomorphic cells and/or associated inflammatory cells, and increased permeability of the stroma lead to the imaging feature.
\end{abstract}

\section{KEYWORDS}

\section{PHAT; FDG-PET; Sarcoma}

\section{Introduction}

Pleomorphic Hyalinizing Angiectatic Tumor (PHAT), first described by Smith et al. in 1996 [1], is a rare lowgrade mesenchymal neoplasm that most often arises in the suprafascial subcutaneous areas of the extremities, and less frequently in the musculature of the limbs. Fewer than 100 cases have been described in the literature. Clinical features are slow-growing, non-metastasizing, locally infiltrative border, and borderline malignancy with a high rate of local recurrence. PHAT describes a heterogeneous group of neoplasms of undetermined tumorigenesis, characterized by clusters of ectatic, fibrinlined, and thin-walled vessels that are surrounded by spindled or pleomorphic neoplastic cells with variable inflammatory components. The neoplastic cells frequently have intracytoplasmic hemosiderin granules, intranuclear inclusions, and low mitotic activity [2-5]. Based only on the morphological findings of histopathology, the biological potential of the tumor is indeterminate. The level of fluorodeoxyglucose (FDG) uptake during positron ${ }^{*}$ Corresponding author. emission tomography (FDG-PET) has been used as an in vivo method for identifying tumor metabolism and biological activity, as well as a prognostic indicator for malignancy. However, FDG uptake in PHAT has not been adequately described in the literature.

\section{Case Report}

62-yrs-old male suffered from sigmoid colon cancer 18 years ago (aged 44, in 1995). A left inguinal hernioplasty with mesh support was performed simultaneously during the surgical resection of the tumor. Afterward, he received 5-FU adjuvant chemotherapy without local radiotherapy. A FDG-PET follow-up examination was performed in 2004, during which a focal area in the left inguinal region adjacent to the urinary bladder displayed increased FDG activity (Figures 1(a1) and (b1)). The maximum standard uptake value (SUVm) of the lesion was 3.2. Magnetic resonance imaging (MRI) showed a tumor in the corresponding region (Figure 2(a1) through Figure 2(a4)). A fixed, firm mass was detected by manual palpation of the left inguinal area. No other symp- 


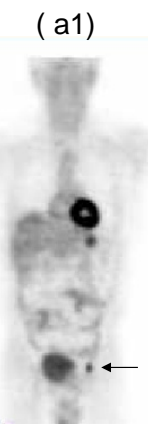

Sep. 2004 (a2)

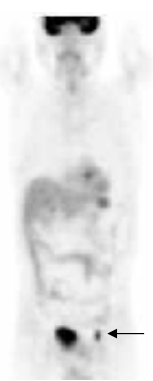

Mar. 2006 (a3)

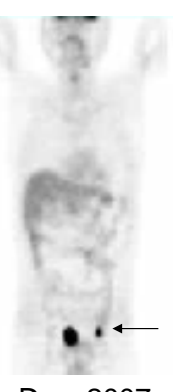

(b1)

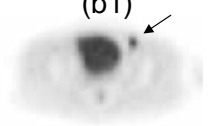

(b2)

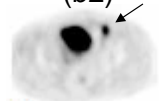

(b3)

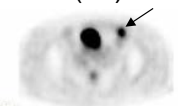

Figure 1. Fluorodeoxyglucose uptake during positron emission tomography (FDG-PET) imaging of the pleomorphic hyalinizing angiectatic tumor (PHAT). FDG-PET was performed in September 2004, and revealed a focal area with increased activity in the left inguinal region (a1). The maximum standard uptake value (SUVm) of the lesion was 3.2. Follow-up FDG-PET in March 2006 (a2) and December 2007 (a3) showed similar findings, with SUVm values of 4.1 and 4.2, respectively. Corresponding images of a transaxial section are shown, from top to bottom (b1, b2 and b3). In addition, the SUVm of the lesion during 3-hr delayed-phase imaging showed no significant changes, compared with the early-phase images. Arrows indicate the lesion.

$$
\text { (a1) }
$$

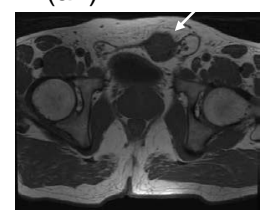

(a4)

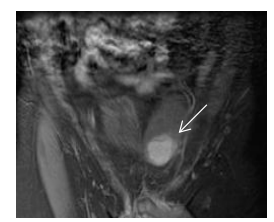

(a2)

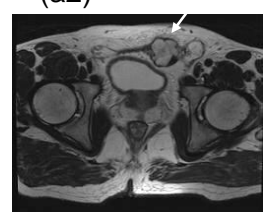

(b)

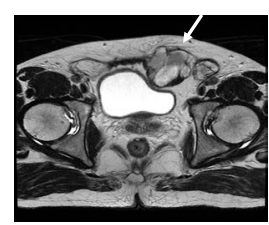

( a3)

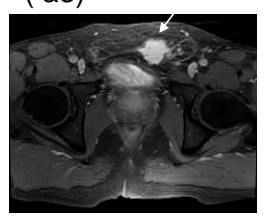

\section{政}

(Figures 1(a2) and (b2)) and (Figures 1(a3) and (b3)), and the follow-up MRI results that showed the size and the feature of the mass had remained unchanged (data not shown). No further signs or symptoms were observed during the remainder of the 17-year follow-up period (1995-2012). Bilateral hernioplasty was received on March 2012 because the symptoms of inguinal hernia had become exaggerated. During the accompanying MRI examination, changes in the size and contour of the inguinal lesion were noted (Figure 2(b)). Fordyce angiokeratoma was also diagnosed based on a number of purple papules on the scrotum, which is often associated with thrombophlebitis of the scrotum, varicocele, and inguinal hernia [6]. During laparotomy, a non-granulomatous tumor was surgically resected from the left rectus abdominis muscle. Gross examination of the tumor showed that it was well-circumscribed, lobulated, solid, and firm (Figure 3(a)), and the histopathology indicated that it was PHAT (Figures 3(b) and (c)). Up to now, he has been robust during one and half years of follow-up.

\section{Discussion}

FDG-PET is a novel modality for metabolic imaging in clinical oncology. It was first used to differentiate benign and malignant lung tumors. However, the specificity of FDG-PET for estimating malignancy remains uncertain. Infection, inflammation, and certain benign pathological variants can mimic the FDG-uptake properties observed in malignancy, contributing to the misinterpretation of FDG-PET images [7]. Quantitative SUVm analysis of tumors is often recommended to discriminate between benign and malignant masses. However, cutoff values for malignancy have not been well defined, and considerable overlap between the SUVm of benign and malignant tumors has been observed. We previously reported the FDG-PET characteristics of three types of benign lesions. These consisted of an angiolipoma in the lower abdominal wall [8], a neurofibroma arising from para-spinal soft tissue [9], and a granuloma formed as a post-opera- (a)

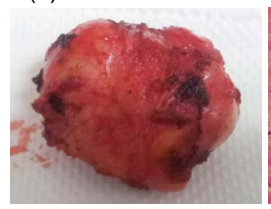

(b)

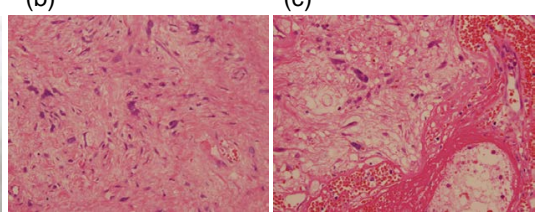

Figure 3. Gross examination and histology of the intramuscular tumor. (a) Grossly, the tumor was encapsulated, wellcircumscribed, lobulated, solid and firm, and no adjacent organ invasion was observed. Microscopic examination (b) and (c) showed hypocellular spinal-cell proliferation, prominent nuclear pleomorphism, low mitotic figure, and ectatic, fibrin-lined, and hyalinized thin-walled with some thrombosed vessels. Weakly positive EMA staining was noted in the immunohistochemical studies. 
tive foreign body in the abdominal cavity [10]. We posited that inflammation, fibrin thrombi, and an ectatic, congestive vascular space had led to an increased accumulation of FDG (SUVm = 3.2) within the angiolipoma. The proliferative spindle cells of the neurofibroma and the intense inflammation associated with the granuloma were considered to have contributed to the increased FDG uptake (SUVm $=4.6$ and 10.8, respectively) in those cases. Only one previous case of PHAT had been found at our institution [3], and that tumor had displayed mild FDG uptake (SUVm = 1.4) during FDG-PET examination. In PHAT, we propose that FDG accumulation in the ectatic vessel, increased uptake of the pleomorphic cells and/or associated inflammatory cells, and increased permeability of the stroma lead to the imaging feature. Whether high SUVm represents sarcomatous transformation of soft tissue neoplasms requires further correlation analysis with histopathological features and clinical outcomes. Folpe and Weiss proposed that PHAT is a neoplasm, rather than a reactive lesion [4]. The occurrence of PHAT in the inguinal area is rare [11], and the tumorigenesis in my case was unclear. However, the reaction of the tissues to surgical manipulation and/or the surgical mesh warrants consideration. The diagnosis of PHAT using MRI alone is unreliable. The features are variable, and notice should be taken when encountering an enhancing subcutaneous tumor with ill-defined margins [12].

\section{REFERENCES}

[1] M. E. Smith, C. Fisher and S. W. Weiss, "Pleomorphic Hyalinizing Angiectatic Tumor of Soft Parts. A LowGrade Neoplasm Resembling Neurilemoma,” American Journal of Surgical Pathology, Vol. 20, No. 1, 1996, pp. 21-29. http://dx.doi.org/10.1097/00000478-199601000-00002

[2] Q. Ke, Erbolat, H. Y. Zhang, H. Bu, S. Li, D. N. Shi, et al., "Clinocopathology Features of Pleomorphic Hyalinizing Angiectatic Tumor of Soft Parts," Chinese Medical Journal, Vol. 20, No., 2007, pp. 876-881.

[3] H. C. Peng, M. T. Huang, D. J. Chen, T. K. Leung and J. S. Chu, "Pleomorphic Hyalinizing Angiectatic Tumor of Soft Parts,” Journal of the Formosan Medical Association, Vol. 109, No. 8, 2010, pp. 616-620.
http://dx.doi.org/10.1016/S0929-6646(10)60100-3

[4] A. L. Folpe and S. W. Weiss, "Pleomorphic Hyalinizing Angiectatic Tumor: Analysis of 41 Cases Supporting Evolution from a Distinctive Precursor Lesion,” American Journal of Surgical Pathology, Vol. 28, No. 11, 2004, pp. 1417-1425.

http://dx.doi.org/10.1097/01.pas.0000141406.02581.fb

[5] S. Wei, Z. Pan, G. P. Siegal, T. S. Winokur, A. J. Carroll and D. Jhala, "Complex Analysis of a Recurrent Pleomorphic Hyalinizing Angiectatic Tumor of Soft Parts," Human Pathology, Vol. 43, No. 1, 2012, pp. 121-126. http://dx.doi.org/10.1016/j.humpath.2011.02.023

[6] M. Machan and V. Konkovic-Capin, "Fordyce’s Angiokeratomas," The New England Journal of Medicine, Vol. 366, 2012, p. 1240. http://dx.doi.org/10.1056/NEJMicm1104749

[7] S. Basu, T. Chryssikos, S. Moghadam-Kia, H. Zhuang, D. A. Torigian and A. Alavi, "Positron Emission Tomography as a Diagnostic Tool in Infection: Present Role and Future Possibilities,” Seminars in Nuclear Medicine, Vol. 39, No. 1, 2009, pp. 36-51. http://dx.doi.org/10.1053/j.semnuclmed.2008.08.004

[8] C. H. Hsu, C. M. Yang and C. H. Cheng, "Angiolipoma Detected by F-18 FDG Positron Emission Tomography,” Clinical Nuclear Medicine, Vol. 28, No. 7, 2003, pp. 604605.

http://dx.doi.org/10.1097/00003072-200307000-00019

[9] C. H. Hsu, C. M. Lee, F. C. Wang and C. L. Fang, "Neurofibroma with Increased Uptake of F-18-Fuloro-2 Deoxy-D-Glucose Interpreted as a Metastatic Lesion," Annals of Nuclear Medicine, Vol. 17, No. 7, 2003, pp. 609-611. http://dx.doi.org/10.1007/BF03006677

[10] C. H. Hsu, C. M. Lee and S. Y. Lin, "Inflammatory Pseudotumor Resulting From Foreign Body in Abdominal Cavity Detected by FDG PET," Clinical Nuclear Medicine, Vol. 28, No. 10, 2003, pp. 842-844. http://dx.doi.org/10.1097/01.rlu.0000090933.66898.d4

[11] M. Y. Choong, P. Z. Ke, C. Y. Huang and T. S. Li, "Pleomorphic Hyalinizing Angiectatic Tumor in the Inguinal area: A Potential Diagnostic Pitfall,” The American Surgeon Journal, Vol. 78, No. 1, 2012, pp. E45-46.

[12] T. K. Subhawong, A. P. Subhawong, E. A. Montgomery and L. M. Fayad, "Pleomorphic Hyalinizing Angiectatic Tumor: Imaging Finding,” Skeletal Radiology, Vol. 41, No. 12, 2012, pp. 1621-1626. http://dx.doi.org/10.1007/s00256-012-1443-0 